\title{
Haemodynamic monitoring of pericardial effusion with cardiac tamponade in a patient with colorectal
}

\section{cancer}

\begin{abstract}
Metastatic cardiac malignancies mainly come from breast, lung, esophagus and lymphoreticular system. Metastasis from colorectal cancer to the heart or pericardium is seldom reported and only sporadic antemortem cases have been reported. We report an unusual case of malignant pericardial effusion with cardiac tamponade caused by metastatic adenocarcinoma of colon in a 58year old patient and haemodynamic monitoring with lithium dilution cardiac output (LiDCO) method of measuring cardiac output due to prepare patient for surgery of subileus.
\end{abstract}

Keywords: pericardial effusion, cardiac tamponade, haemodinamic monitoring, colorectal cancer
Volume 2 Issue 4 - 2015

Daniela Novosel Matijević
Department of Anesthesiology, Ireland

Correspondence: Daniela Novosel Matijević, Consultant Anaesthetist, Department of Anesthesiology St.Luke $\square$ s Hospital Kilkenny, Kilkenny, Ireland,

Email danielanovosel@hotmail.com

Received: May 05, 2015 | Published: June 04, 2015

\section{Introduction}

Malignant pericardial effusion is a rare and serious manifestation of advanced malignancies. Pericardial effusion defines the presence of an abnormal amount of fluid in the pericardial space. Malignant pericardial effusions are caused by cancer that begins in the pericardium or the heart muscle, or by cancer that has spread there from the esophagus, thymus, or lymph system and are commonly caused by lung cancer in males and breast cancer in females. ${ }^{1-3}$ Pericardial effusions can be acute or chronic, and the time course of development has a great impact on the patient's symptoms. Treatment is directed at both removal of the pericardial fluid and alleviation of the underlying cause which usually is determined by a combination of fluid analysis and correlation with comorbid illnesses. Clinical manifestations of pericardial effusion are highly dependent upon the rate of accumulation of fluid in the pericardial sac and include chest discomfort, dyspnoea, tachycardia, pulsus paradoxus, increased central venous pressure, hypotension and cardiogenic shock, although some patients remain undiagnosed until death. ${ }^{4,5}$ Rapid accumulation of pericardial fluid may cause elevated intrapericardial pressures with as little as $80 \mathrm{~mL}$ of fluid. Slowly progressing effusions can grow to $2 \mathrm{~L}$ without symptoms. ${ }^{6}$ Pericardial effusion is the primary or contributory cause of death in $85 \%$ of cancer patients with symptomatic effusions. ${ }^{7,8}$ Because most patients are asymptomatic, the incidence is much higher in autopsy series. ${ }^{9}$

LiDCO is a minimally invasive indicator dilution technique for the measurement of cardiac output and providing continuous, reliable and accurate assessment of the hemodynamic status of critical care and surgery patients. A small dose of lithium chloride is injected as an intravenous bolus, and cardiac output is derived from the dilution curve generated by a lithium-sensitive electrode attached to the arterial line. Besides cardiac output with LiDCO method we can measure: cardiac index, stroke volume, systemic vascular resistance, intrathoracic blood volume index, oxygen delivery, heart rate and mean blood pressure. ${ }^{10}$

\section{Case report}

A 58year old patient was hospitalized for tumor of final intestine and planned surgical procedure. The colonoscopy findings showed infiltrative process which almost completely closed the interior side of the bowel. During preoperative preparation the patient developed images of subileus. Biopsy proved adenocarcinoma of colorectal cancer. The patient suffered from hypertension and diabetes mellitus type II. During routine preoperative preparation, CT revealed pericardial effusion. Preoperative ultrasound of the heart confirmed minimal pericardial effusion $(17 \mathrm{~mm})$ and left ventricular ejection fraction (EFLV) 55\%. The patient was hemodynamically stable. On the 6th day of hospitalization, the patient suddenly became disturbed, dyspnoic, pale, sweaty, tachycard and hypotensive and was admitted to the intensive care unit. On the admission the patient was hemodynamically unstable, central venous catheter and cannula for arterial blood pressure monitoring were set and LIDCO monitoring was connected. Haemodynamic monitoring with LiDCO method showed that heart stroke volume was $67 \mathrm{~mL}$, cardiac output $6,3 \mathrm{~L} /$ min, systemic vascular resistance $769 \mathrm{dyn} / \mathrm{s} / \mathrm{cm}^{3}$, heart rate $100 / \mathrm{min}$, blood pressure was 100/56 and central venous pressure $11 \mathrm{mmHg}$. Three days after being admitted to intensive care unit, haemodynamic monitoring with LiDCO method noticed a sudden change of values, heart stroke volume has fallen to $22 \mathrm{~mL}$, cardiac output to $2.6 \mathrm{~L} / \mathrm{min}$, blood pressure to $80 / 66 \mathrm{mmHg}$, systemic vascular resistance has risen to $2270 \mathrm{dyn} / \mathrm{s} / \mathrm{cm}^{3}$, heart rate to $119 / \mathrm{min}$ and central venous pressure to $22 \mathrm{mmHg}$. An urgent ultrasound of the heart confirmed a large pericardial effusion of $5 \mathrm{~cm}$, EFLV 33\%, partial diastolic collapse of the front wall of the right ventricle and right atrial systolic collapse, which showed cardiac tamponade. An urgent pericardiocentesis was done, and through the placed catheter it was received a $560 \mathrm{~mL}$ of hemorrhagic, cytologically proven malignant content. The patient became haemodynamically stable with inotropic support with dobutamine $(6 \mathrm{mg} / \mathrm{kg} / \mathrm{min})$, prepared for palliative surgery and planned surgical procedure was done.

\section{Discussion}

Malignant pericardial effusion is a potentially fatal complication of malignancy and very rarely assosiated with colorectal cancer. Chen JL et al. ${ }^{11}$ report an unusual case of malignant pericardial effusion and subsequent tamponade that was the earliest manifestation caused by metastatic adenocarcinoma of colon. Ben Yosef $\mathrm{R}$ et al. ${ }^{12}$ present a patient who developed malignant pericardial effusion following 
surgery. Malignant pericardial effusion is frequently asymptomatic and usually is detected incidentally. Symptomatic cases, however, often manifest with cardiac tamponade, which can rapidly lead to cardiovascular collapse and death unless promptly treated. Pericardial effusion can lead to cardiac tamponade which is a life threatening condition and accurate diagnosis and prompt intervention are necessary. Cardiac tamponade refers to cardiac compression caused by abnormal accumulation of pericardial fluid which limitates ventricular filling and reduces stroke volume and cardiac output. ${ }^{13}$ Clinical diagnosis of malignant pericardial effusion is difficult, and can be easilly confused with other conditions such as pulmonary embolism and silent myocardial infarction. Cardiac tamponade is often difficult to diagnose without performing echocardiography.

In our case, a spectrum of abnormal haemodynamic changes associated with this condition has been identified with LIDCO method and raised the suspicion of a cardiac tamponade which was confirmed by echocardiography. The use of LIDCO method significantly reduced the time period of diagnosis of acute deterioration of pericardial effusion and thus prevented the occurrence of fatal pericardial tamponade. By examining the available literature no description of using LIDCO methods in hemodynamic monitoring of pericardial effusion and cardiac tamponade was found.

\section{Conclusion}

Because malignant pericardial effusion is a rare complication of colorectal cancer, it is harder to raise a suspicion of cardiac tamponade in hemodynamically unstable patients. In our case haemodynamic assessment with LIDCO method provided a rapid resolution of this fatal complication. LIDCO method could be a complementary technique for precise assessment of haemodynamic impact of malignant pericardial effusion in haemodinamically unstable patient in intensive care unit.

\section{Acknowledgements}

None.

\section{Conflicts of interest}

The authors declare that there are no conflicts of interest.

\section{Funding}

None.

\section{References}

1. Cozzi S, Montanara S, Luraschi A, et al. Management of neoplastic pericardial effusions. Tumori. 2010;96(6):926-929.

2. Lokich JJ (1973) The management of malignant pericardial effusions. JAMA. 1973;224:1401-1404.

3. Dempke W, Firusian N. Treatment of malignant pericardial effusion with 32P-colloid. Br J Cancer. 1999;80(12):1955-1957.

4. Minagawa T, Murata Y, Uchikawa S, et al. Malignant pericardial tamponade in a patient with hormone-refractory prostate cancer. Int $J$ Clin Oncol. 2010;15(1):101-103.

5. Kim SH, Kwak MH, Park S, Ket al. Clinical characteristics of malignant pericardial effusion associated with recurrence and survival. Cancer Res Treat. 2010;42(4):210-216.

6. Van Steijn JH, Sleijfer DT, Van der Graaf WT, et al. How to diagnose cardiac tamponade. Neth J Med. 2002;60(8):334-338.

7. Buzaid AC, Garewal HS, Greenberg BR. Managing malignant pericardial effusion. West J Med. 1989;150(2):174-179.

8. Thurber DL, Edwards JE, Achor RW. Secondary malignant tumors of the pericardium. Circulation. 1962;26:228-241.

9. Adenle AD, Edwards JE. Clinical and pathologic features of metastatic neoplasms of the pericardium. Chest. 1982;81(2):166-169.

10. Pinsky MR, Payen D. Functional hemodynamic monitoring. Update in Intensive Care and Emergency Medicine. Cardiology \& Angiology. 2005;42:183-192.

11. Chen JL, Huang TW, Hsu PS, et al. Cardiac tamponade as the initial manifestation of metastatic adenocarcinoma from the colon. Heart Surg Forum. 2007;10(4):E329-330.

12. Ben Yosef R, Warner E, Gez E, et al. Malignant pericardial effusion associated with metastatic rectal cancer. J Chemother. 1989;1(5):342345 .

13. van Steijn JH, Sleijfer DT, van der Graaf WT, et al. How to diagnose cardiac tamponade. Neth J Med. 2002;60(8):334-338. 